\title{
The Multi-Breakage Phenomenon in Air Bending Process
}

\author{
Vitalii Vorkov ${ }^{1, a^{*}}$, Richard Aerens ${ }^{\mathrm{b}}$, Dirk Vandepitte ${ }^{1, \mathrm{c}}$ and Joost R. Duflou ${ }^{1, \mathrm{~d}}$ \\ ${ }^{1}$ Department of Mechanical Engineering, KU Leuven, Belgium \\ avitalii.vorkov@mech.kuleuven.be, ${ }^{b}$ richard.aerens@hotmail.com, \\ cdirk.vandepitte@mech.kuleuven.be, djoost.duflou@mech.kuleuven.be
}

Keywords: multi-breakage phenomenon, springback, high-strength steel, finite-element modeling, air bending

\begin{abstract}
In this work, the multi-breakage effect has been studied by means of an experimental campaign and finite element analysis. We suggest that large radius bending (XL-bending) consists of three phases that are distinguishable according to the type of contact of the plate with the tool: 1-point, surface and 2-points. In the experimental investigation the high-strength steel Weldox 1300 and a $40 \mathrm{~mm}$ radius punch were used. The authors created a camera setup to film the multi-breakage effect. Additionally, finite-element calculations were performed to confirm the hypothesis of the three phases of the bending process. For the springback and the bending force evaluation, the difference in the moment distribution for each phase has been calculated in the case of a beam. It shows that the multi-breakage effect must be taken into account to obtain a good accuracy for the springback and the bending force calculation.
\end{abstract}

\section{Introduction}

Conventional (or small radius) bending is a well-known and predictable technology which can be described by a traditional 3-point bending model. If however a part is bent with a punch having a radius larger than $3 \ldots 4 \ldots 5$ times (depending on the material) the thickness of the plate, the progress of the bending process is quite different, with its own specific aspects. The multi-breakage phenomenon lends its name to the discontinuous contact surface between punch and plate (see Fig. 1): the contact surface splits or "breaks". This kind of behavior is best described by 4-point bending and causes the actual bend radius to be smaller than the punch radius.

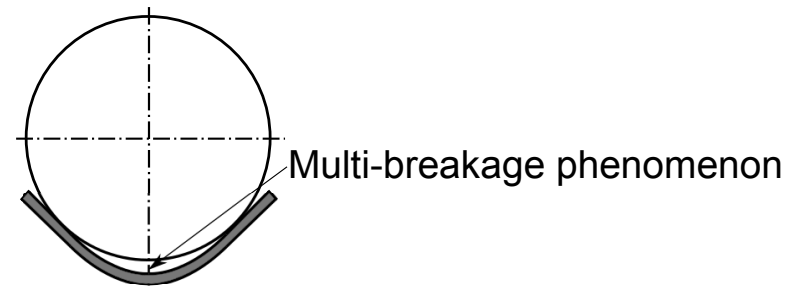

Fig. 1 - Scheme of the multi-breakage effect: splitting or „breaking“ of the contact surface

The calculation of springback is a very challenging task, because many factors affect the part during and after bending [1]. The multi-breakage effect takes place in almost every material and the phenomenon causes errors in the calculations if the change in moment distribution is not taken into account. Despite the importance of large radius bending, there is a lack of information about this phenomenon. The information available in the Internet, e.g. [2], in books, e.g. [3], and scientific literature, e.g. [4], provides only general information or results of measurements for one specific material and one angle (usually $90^{\circ}$ ).

The XL-bending process can be divided into 3 phases as depicted in Fig. 2. The first phase is an initial contact or 1-point contact interaction. Subsequently, surface contact takes place: the punch and the plate interact over a continuous contact surface. The final phase occurs after splitting of the contact surface and the contact becomes discontinuous. 

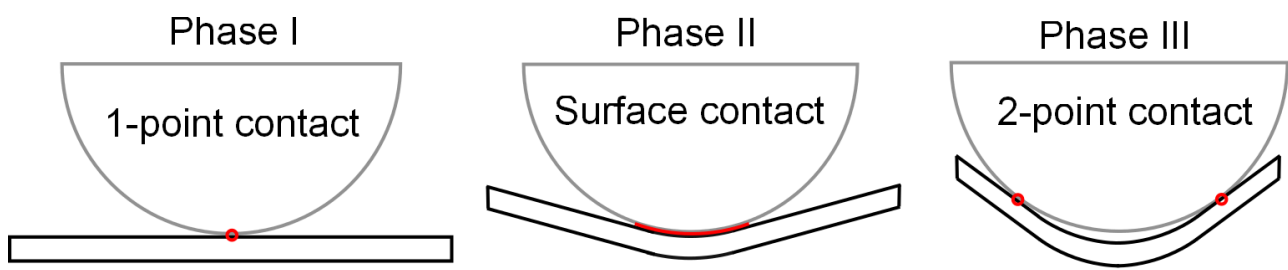

Fig. 2 - Typical XL-bending phases

The aim of the work is to explain the importance of the multi-breakage effect in the large radius bending process of high-strength steel; construction steel has also been used in the experimental investigation for comparison. We used the experimental approach that allows visualization of the multi-breakage effect.

\section{Experimental investigation}

The authors performed experiments on a press-brake with a capacity of 50 tons. The bending angle was $90^{\circ}$. High-strength steel Weldox 1300 by SSAB [6] and construction steel St37 (S235JR) were processed in 4-mm thick plates of 200 by $150 \mathrm{~mm}$. All material were used "as is" without additional treatment except cutting to abovementioned dimensions. A sketch of the tooling is given in Fig. 5.
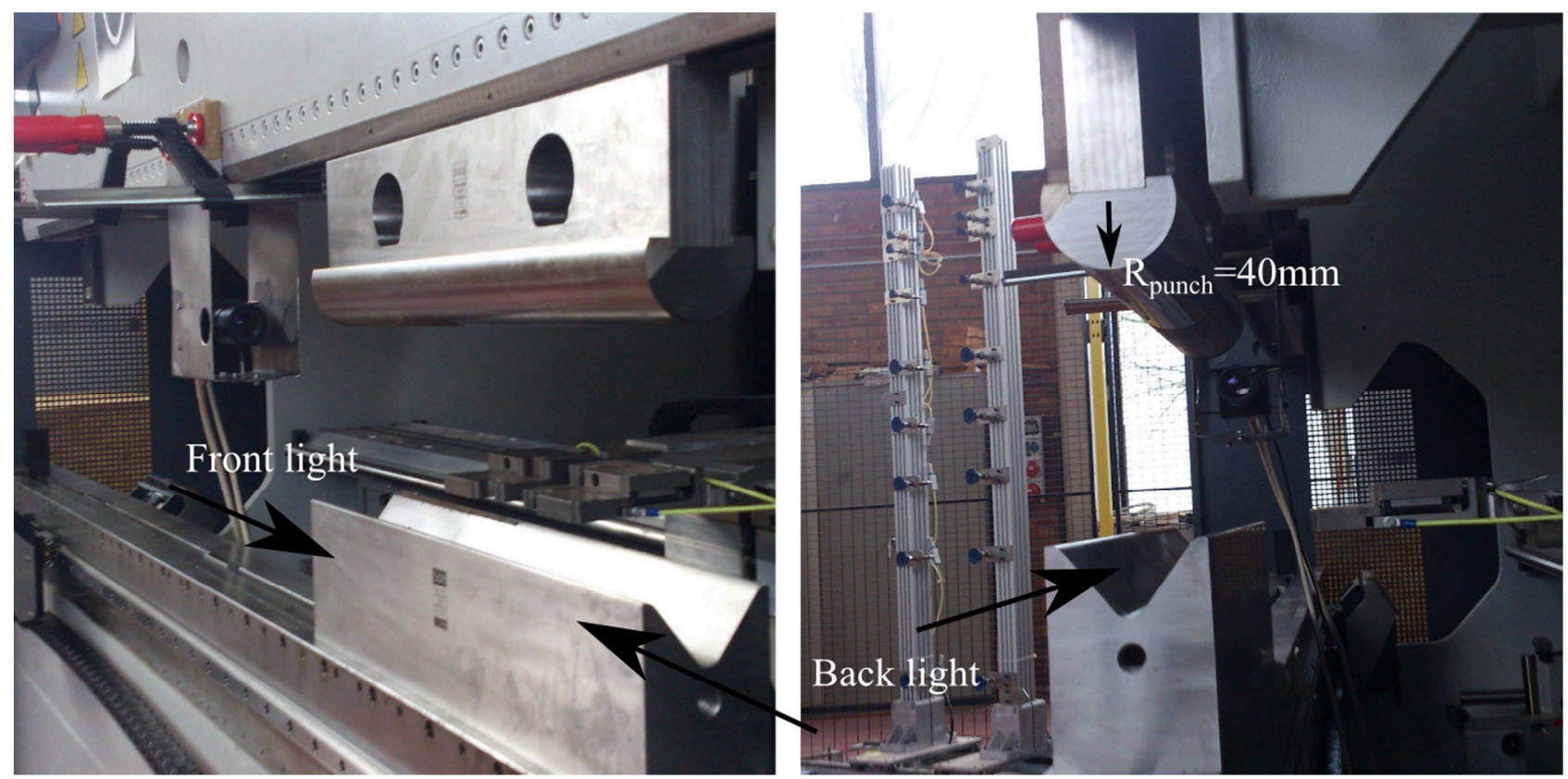

Fig. 3 - Overview of experimental setup

For the experimental investigation we developed a special camera setup (see Fig. 3). The setup consists of a camera fixed on the ram of the press-brake and the LabVIEW program. For filming the bending process the authors used a high-speed camera. The camera was positioned in such a way that the axis of the lens pointed to the lowest position of the punch. In order to create pictures with visible multi-breakage effect, usage of additional sources of light is mandatory. In the experiments we used a strong source of light from the side of the camera (front light) and a secondary source from the back side (back light).

The results of the experimental investigation are shown in Fig. 4; "the light zones" between the plate and the punch bring out the multi-breakage effect. The multi-breakage effect is pronounced during bending for both materials, St37, and Weldox 1300. As we can see in Fig. 4 a, b, the effect is more pronounced for St37. The reason for this could be the higher strength of Weldox 1300 or its lower hardening exponent $n$ (for St37 n=0.14...0.19 [8] while for Weldox, $n=0.06$ [6]). 


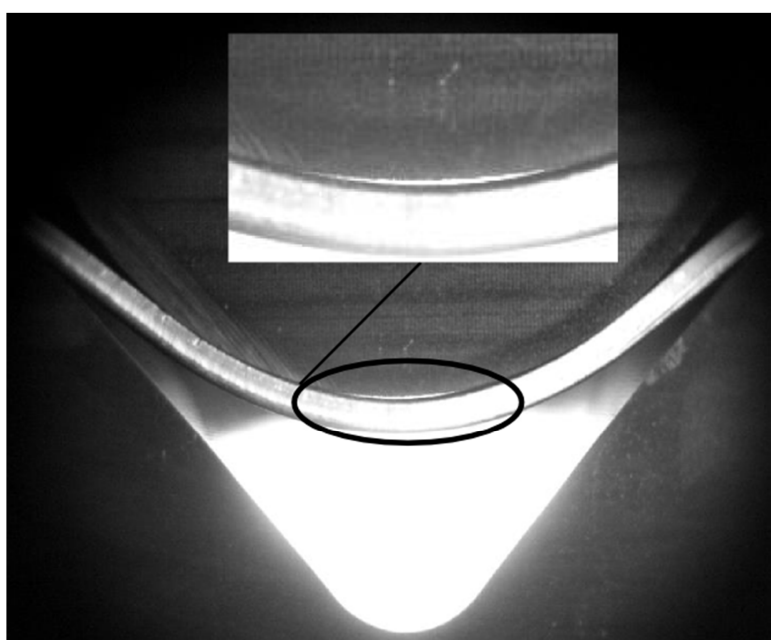

a) the intermediate state with the largest gap for St37

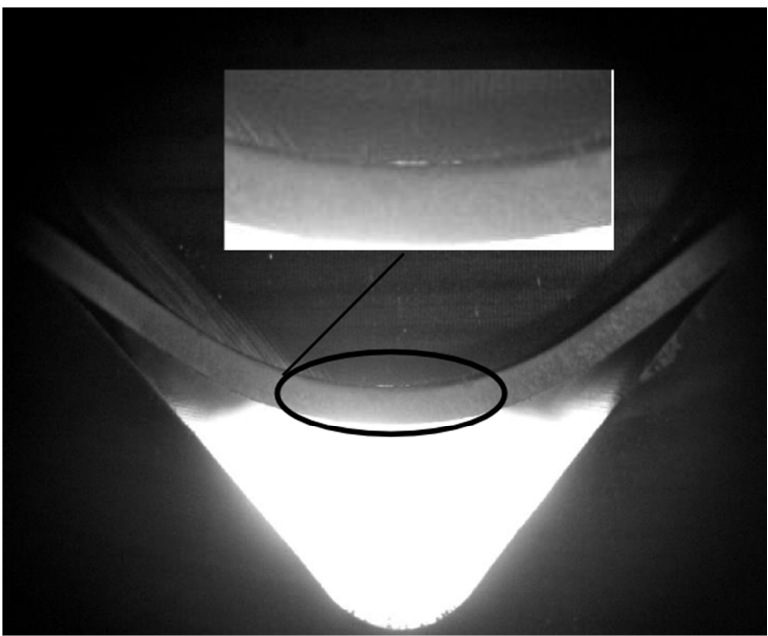

c) the intermediate state with the largest gap for Weldox 1300

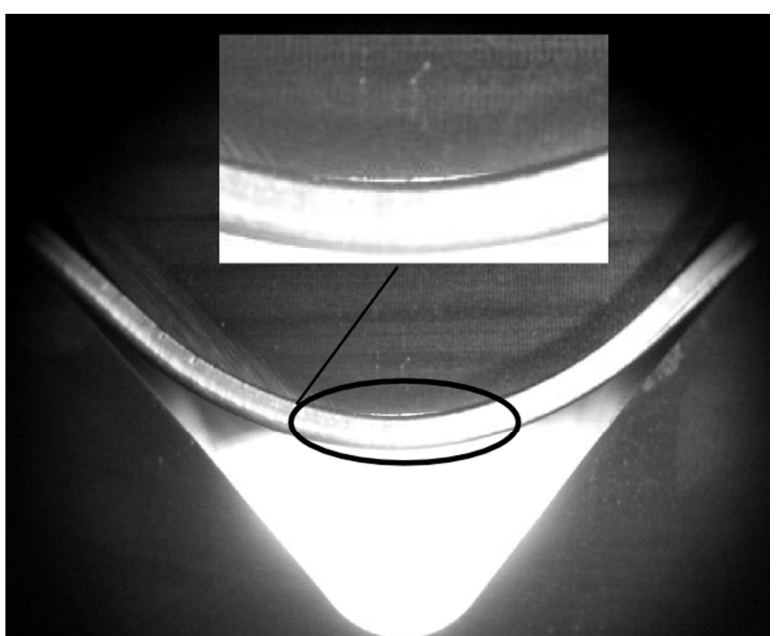

b) the final state of bending for St37

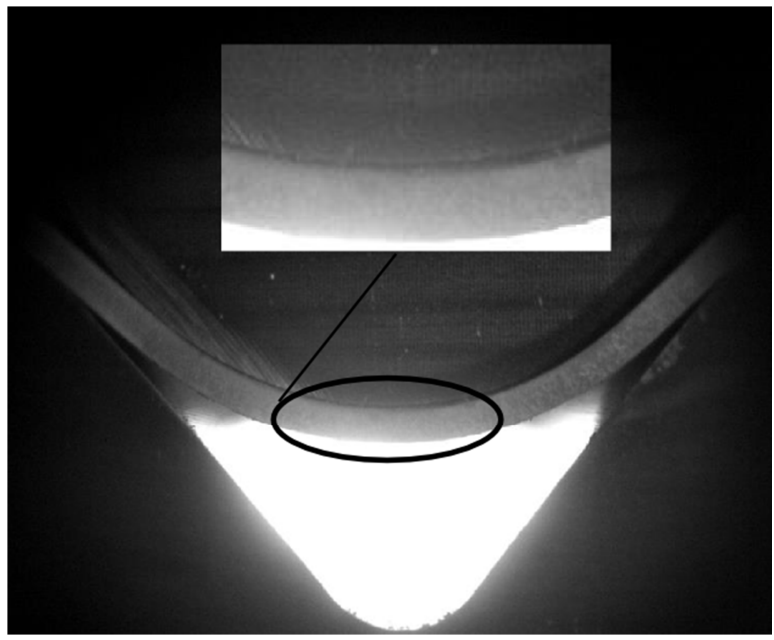

d) the final state of bending for Weldox 1300

Fig. 4 - Experimental investigation of the multi-breakage effect a) and b) St37,

c) and d) Weldox 1300 - ,the light zones" between the punch and the plate represent the split in the contact surface or the multibreakage effect

\section{Finite-element investigation}

In the present work we used the Abaqus software with an implicit solver to simulate the bending of the 4-mm plate made of Weldox 1300. The finite-element model is shown in Fig. 5. Since Weldox 1300 was the target material in this study, all finite-element calculations involve only this high-strength material. The dimensions of the plate for finite element simulation are the same as for the experimental investigation.

The tooling (the punch and the die) were modeled as 3-D rigid surfaces, the vertical movement of the punch is $\Delta Y=22 \mathrm{~mm}$. The tooling and the plate have the same length. A shell element with 7 integration points through the thickness has been selected. The plate is modeled with four-node plane strain shell elements (CPE4R) [5]. 70 elements are distributed along the width with concentration towards the center of the plate with ratio 1:5, and the thickness of the elements is distributed symmetrically to middle line (see Fig. 5).

For the material, we implemented an isotropic Hollomon hardening model (see Eq. 1) with parameters from Table 1. As yield locus, we selected Von Mises. The Coulomb friction model was applied with a friction coefficient 0.2 between tools and plate. 
$\sigma=K \cdot \varepsilon^{n}$

Where:

$\varepsilon$ : equivalent plastic strain

$\mathrm{K}$ : strength coefficient

$\mathrm{n}$ : hardening exponent

Table 1 - Material properties of Weldox 1300 [6]

\begin{tabular}{cccccc}
\hline $\begin{array}{c}\text { Density } \\
{\left[\mathrm{kg} / \mathrm{m}^{3}\right]}\end{array}$ & $\begin{array}{c}\text { Poisson's } \\
\text { ration } \mu[-]\end{array}$ & $\begin{array}{c}\text { Initial elasticity } \\
\text { modulus E } \\
{[\mathrm{MPa}]}\end{array}$ & $\begin{array}{c}\text { Plane strain } \\
\text { elasticity } \\
\text { modulus E' } \\
{[\mathrm{MPa}]}\end{array}$ & $\mathrm{K}[\mathrm{MPa}]$ & $\mathrm{n} \mathrm{[-]}$ \\
\hline 7800 & 0.3 & $2 \mathrm{e} 5$ & $2.2 \mathrm{e} 5$ & 1770 & 0.06 \\
\hline
\end{tabular}

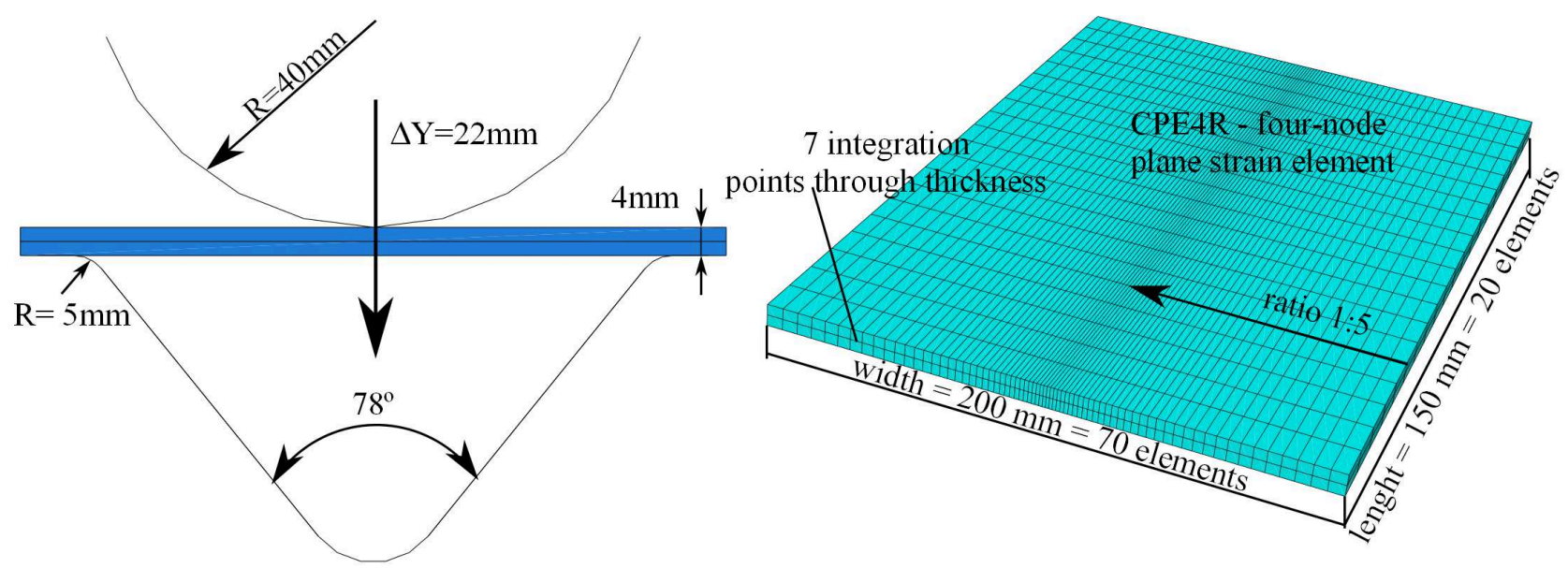

Fig. 5 - Overview of loading scheme and finite-element mesh

Fig. 6 presents the results (contact pressure on the plate) of the finite-element computations. One can distinguish clearly the three phases: a) 3-point bending, b) surface contact bending, c) and d) 4-point bending. The first two phases are shorter than the 4-point bending phase. The assumption of a "contact surface" is valid only at the very beginning of phase 2 since the simulation shows that in the contact zone the contact pressure is mainly distributed close to the border of the contact surface (Fig. 6b). Difference in contact pressure along the plate is due to presence of the anticlastic effect in the shell model. In fact, instead of the surface loading, the 2-point contact with the plate (4-point bending) follows directly after the initial contact. 


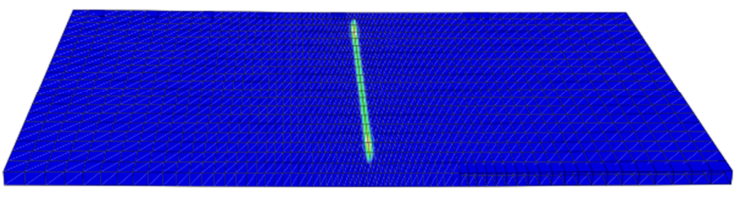

a) Initial contact

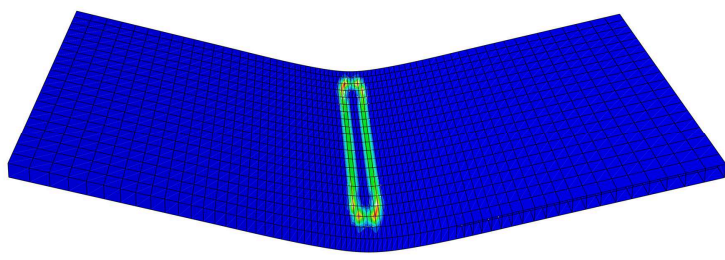

c) Initial 2-point contact

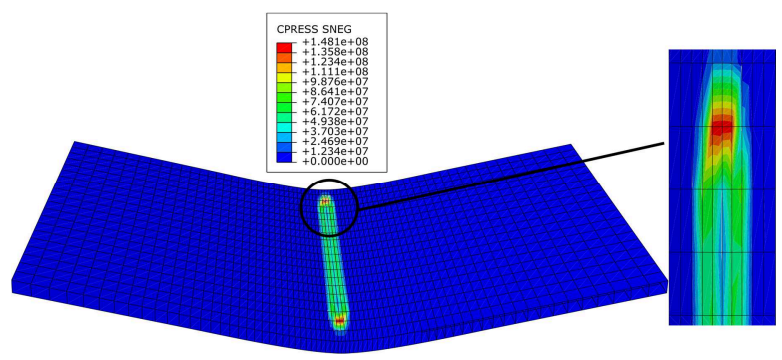

b) Surface contact

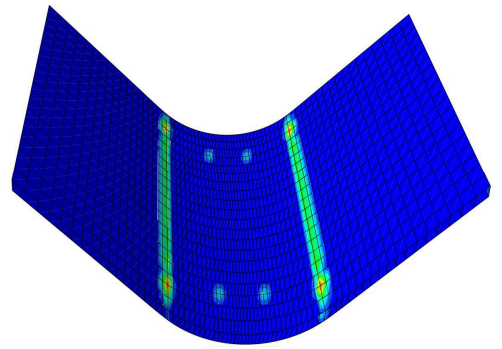

d) Final 2-point contact

Fig. 6 - Results of the finite-element calculations

\section{Influence of the multi-breakage on the springback and the bending force}

The finite element solution is handy to get the full strain-stress distribution in a plate during bending, but finite element calculations are time consuming. An analytical model is the only one that can be used for control purposes in real manufacturing processes. An example of this kind of processes is adaptive bending [7].

In order to estimate the significance of the multi-breakage phenomenon, Eq. 2 for the springback angle calculation $\Delta \beta[8]$ has been used.

$\frac{\Delta \beta}{2}=\frac{1}{E^{\prime} I} \int_{0}^{l_{t o t}} M d s$

Where

$\beta=$ springback angle

$\mathrm{E}^{\prime}=$ plane strain elasticity modulus

$\mathrm{I}=$ second moment of area

$\mathrm{M}=$ local bending moment

$\mathrm{s}=$ abscissa on the curvilinear axis, coinciding with the neutral axis of the beam

$l_{\text {tot }}=$ length of a half beam

The equivalent elastic modulus is used to take into account the plane strain condition, which is the case in bending of large plates. The equivalent elastic modulus is calculated as follows:

$E^{\prime}=\frac{E}{1-\mu^{2}}$

We assumed a beam hypothesis to get a quick estimation of the multi-breakage effect. As we discussed previously we considered three phases of the bending: 1-point contact, surface contact, 2-point contact and the corresponding moment calculations for each phase are depicted in Table 2.

The results of the calculations according to Eq. 2 given in Table 2 show the difference in springback calculation between the conventional bending (3-point bending) and XL-bending 
(4-point bending). The quantitative difference depends on each particular case, but the larger the punch radius, the larger the value of "a" (see Table 2) what leads to a more pronounced difference. This underlines the importance of knowing the exact position of the contact points for the calculation of the springback in an analytical model.

The beam theory says that in the region with increasing moment, the radius of curvature should decrease, but in case of a surface contact, this decrease is impossible. One of the possible origins of the multi-breakage effect could be the impossibility for the plate to fit the loading scheme and the plate changes its shape to balance the increasing of the moment.

Table 2 - Difference in moment distribution for each phase of XL-bending

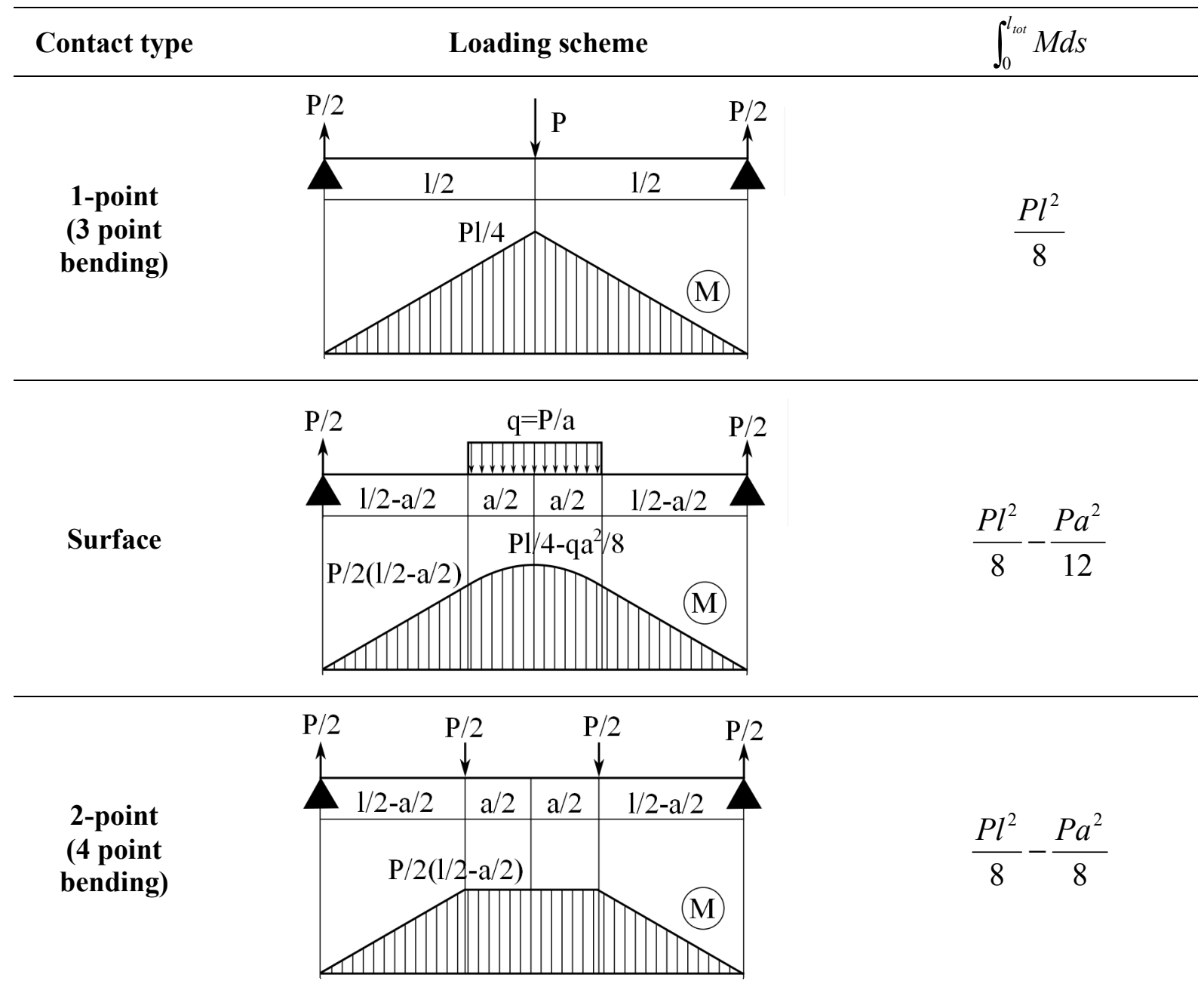

From the last sketch another interesting consequence can be deduced for the bending force P. In order to obtain a prescribed curvature (slightly higher than that of the tool), the material needs an internal bending moment. This moment must be balanced by the external bending moment. So, when the distance "a" increases, the value of $(1 / 2-a / 2)$ will decrease and consequently the value of $P$ must increase (to hold the required moment). It underlines a second time the importance to know the exact position of the contact points for the calculation of the bending force in an analytical model.

\section{Conclusion and discussion}

In order to investigate the multi-breakage phenomenon an experimental approach and finite-element modeling have been used in large radius bending of high strength steels. The experimental investigation shows that this effect is notable as well for a high-strength steel as for construction steel. Finite element calculations - performed in Abaqus - show strong evidence of 
3-phase bending. The analytical investigation shows that, even in a beam approximation, the difference in the loading scheme could lead to a significant error in the springback calculations.

The three distinguished bending phases can be reduced to two phases (1-point contact and then 2-points contact) or even to single phase (immediately 2-points contact, i.e. the so called "4-point" bending including the contact points with the die) depending on the punch radius. Indeed, on one hand, the surface contact phase behaves like 2-points bending, due to the distribution of the contact pressure, which is concentrated near the edge of the contact zone, and on the other hand, the 1-point contact phase is too short to be taken into account. It reasonable to assume 4-point bending from the outset and to calculate the moment distribution according to the position of the contact points. This issue will be the scope of future work.

\section{References}

[1] R.H. Wagoner, H. Lim, M-G. Lee, Advanced issues in springback, International Journal of Plasticity, 45 (2013) 3-20.

[2] Information on http://www.ntlworld.com.

[3] S.D. Benson, Press brake technology, A guide to precision sheet metal bending, Society of Manufacturing Engineers, 1997.

[4] I. Burchitz, Improvement of springback prediction in sheet metal forming, University of Twente, 2008.

[5] Information from Abaqus User's Manual v 6.12.

[6] Information on http://www.ssab.com.

[7] W. Serruys, Adaptive Control in CNC Sheet Metal Processing, Key Engineering Materials, 473 (2011) 13-20.

[8] R. Aerens, Le pliage en l'air, CRIF, Section Construction Mécanique, MC110, 2000. 